\title{
A case of modified advancement flap for an auricular skin defect
}

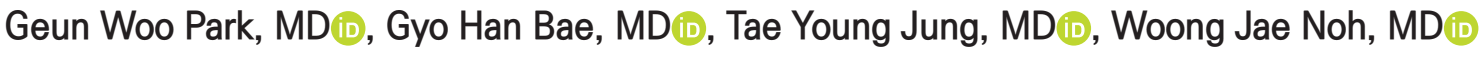 \\ Department of Otorhinolaryngology Head and Neck Surgery, Maryknoll Hospital, Busan, Rep. of Korea
}

The auricle is susceptible to damage because of its position in the body. Reconstruction of auricular defects, especially on the anterior side, is challenging considering the thin skin compartment and complex topography. Various techniques can be used for reconstruction, of which local flaps are preferred owing to their excellent outcomes. We report a case of cutaneous squamous cell carcinoma in the cavum concha that was successfully reconstructed with a modified advancement flap technique. We would like to share our experience and suggest the modified advancement flap as an option for reconstruction.

Keywords: reconstructive surgical procedures; skin neoplasms; surgical flaps

\section{Introduction}

The auricle is susceptible to damage because of its position in the body.

Trauma, iatrogenic excision of neoplasms, and infection can cause auricular defects. Reconstruction of auricular defects, especially those occurring in the concha, is challenging. Various techniques can be used for reconstruction, of which local flaps are predominantly used owing to their excellent functional and esthetic outcomes.

Herein, we described the case of a patient who had an anterior ear skin defect after resection of cutaneous squamous cell carcinoma (cSCC). We modified advanced flap which harvested from the postauricular area to cover the defect in the cavum concha and achieve satisfactory functional and esthetic outcomes.

\section{Case report}

An 88-year-old male patient visited our hospital for management of a right auricular papillomatous mass that had grown rapidly since a month (Fig. 1). SCC was confirmed by punch biopsy, and there was no evidence of lymph node or distant metastasis on enhanced neck computed tomography and torso positron emission tomography-computed tomography.

Surgical resection involving the skin, subcutaneous tissue, and perichondrial layer of the cavum concha was performed under local anesthesia, and negative margins were confirmed histologically. After surgical resection, a skin defect $2 \mathrm{~cm}$ in diameter persisted (Fig. 2A, 3A).

Postauricular modified advancement flap reconstruction was performed after five days of resection surgery to confirm the absence of remnant cancer and negative surgical margins. The pedicle of the full-thickness flap was created in the posterior auricular area at the same level as the defect. A cartilage slit was 
created along the lateral margin of the defect site. The flap was incised superficially to create the skin island, which penetrated the cartilage slit and was moved to the defect site on the anterior surface of the cavum concha (Fig. 3B, C). Primary closure of the flap and defect was done using nylon 5-0 (Fig. 2B, C, 3D).

The flap healed completely, 1 month after the reconstruction and there were no complications such as dehiscence or necrosis (Fig. 4A, B).

\section{Discussion}

cSCC is the second most common skin cancer in Korea [1]. Risk factors for cSCC include old age and exposure to ultraviolet radiation. Generally, cSCC has a good prognosis; however,

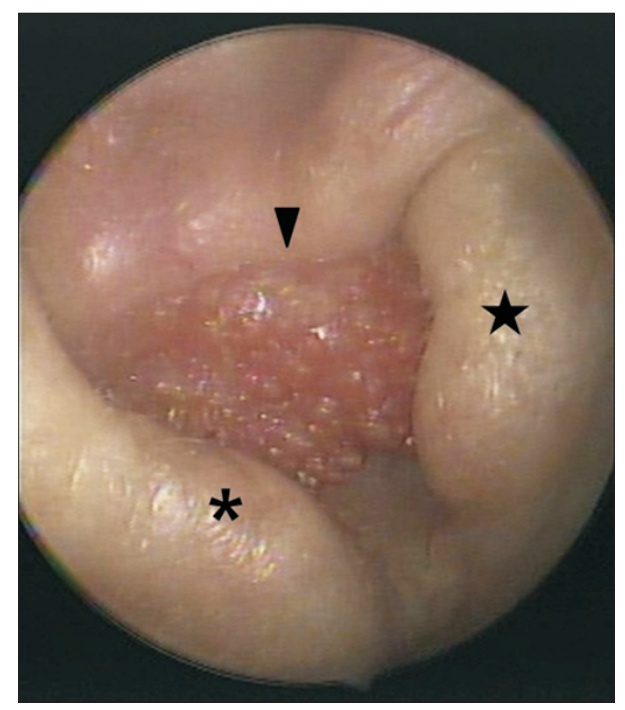

Fig. 1. Gross finding showed papillomatous mass (arrow head) in right cavum concha, which is blocking the orifice of external auditory canal (star: tragus; asterisk: antitragus).

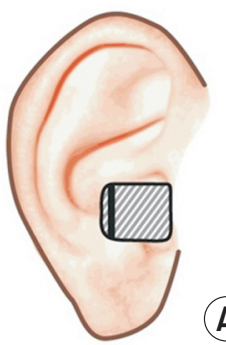

(A)

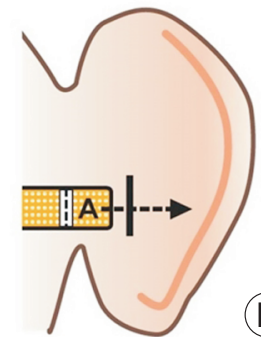

(B) metastasis to regional lymph nodes occurs in $5 \%$ of the cases. More than $50 \%$ of cSCC cases originate from the head and neck, and $8.2 \%$ originate from ear [1]. In the auricle, cSCC commonly occurs on the helix, antihelix, and posterior ear, and less frequently on the tragus, lobule, or conchal cavity [2].

As cSCC consists of atypical keratinocytes invading the dermis, the primary treatment is surgical resection with a free resection margin [3]. A study defined high-risk lesions as tumors with diameter $\geq 2 \mathrm{~cm}$, higher histologic grade, invading subcutaneous tissue, and those located in the eyelids, ears, nose, and lips [4]. High-risk lesions must be resected surgically with a resection margin of at least $6 \mathrm{~mm}$ [4]. Mohs micrographic surgery was introduced for complete removal of cancer, and has demonstrated enhanced surgical outcomes than the original surgical resection technique, owing to better preservation of normal tissue. Tissue preservation is important in the facial area considering both functional and cosmetic aspects. Therefore, Mohs micrographic surgery can be an indication for cancers in
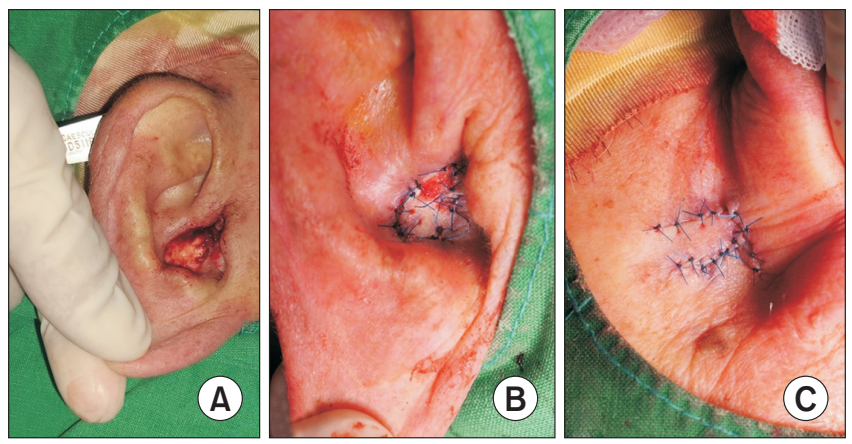

Fig. 2. A large skin defect in the cavum concha remained after resection of cancer (A). In second-stage operation, postauricular advancement flap technique was used for reconstruction of cavum concha defect (B, C). Both flap (B) and postauricular area where the flap was harvested $(C)$ had primary skin closure by nylon 5-0.

Fig. 3. Scheme of the advancement flap technique used for anterior auricle defect reconstruction. Skin defect which revealed cartilage (shaded area) at cavum concha. Slit was made by removal of cartilage at lateral margin of defect (marked as thick black line) (A). Flap was harvested from postauricular area (yellow dotted area) and incised superficially (white area with dotted line in the middle) to make skin island (area marked with A) (B). Flap penetrated the cartilage slit (marked as arrow) (B, C) and transferred from postauricular area to anterior auricular defect area (C). The flap conceals defect and primary suture of the flap and donor site was done (D). 

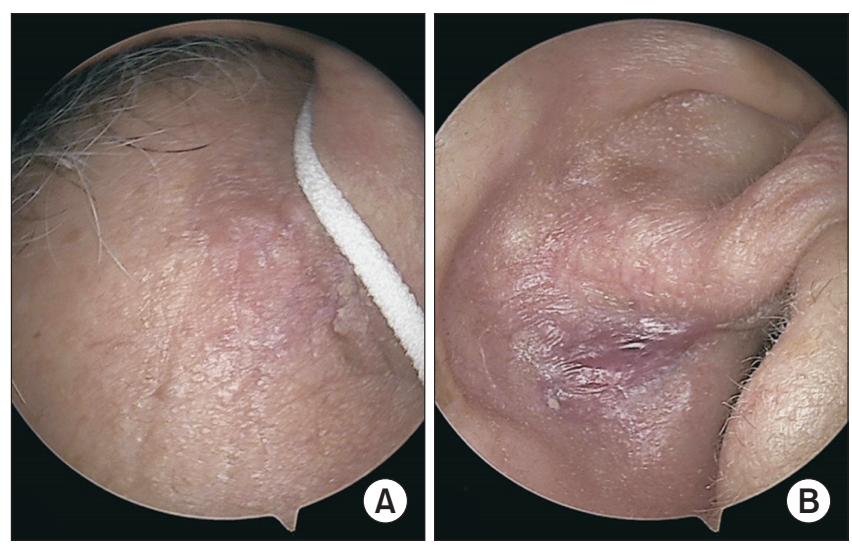

Fig. 4. One month after second-stage operation, anterior auricular area where flap was transported (A) and postauricular area where the flap was made (B) showed complete recovery and no complication such as dehiscence and necrosis.

the facial area [5].

When cSCC occurs on the anterior aspect of the auricle, there is a high possibility of a large defect following surgical excision. Reconstruction of large defects is challenging due to the complex topography of the ear and thin skin layer; however, it is important because secondary healing can lead to poor cosmetic outcomes, chondritis, or infection [3]. Surgical options include skin grafting, regional flaps such as full-thickness skin grafts obtained from the clavicle or counterpart postauricular area, and local flaps, such as preauricular transposition flap, tubed pedicle flap, postauricular pull-through flap, and postauricular revolving door island flap [6,7]. Among these techniques, surgeons commonly prefer local flaps, as they provide better cosmetic and functional outcomes. Furthermore, local flaps are easier to perform and require less surgical time compared to other techniques [7].

The postauricular island flap, also known as the postauricular revolving door island flap, was first introduced by Masson in 1972 [6]. In this technique, the flap is harvested from the postauricular and mastoid areas, each of which constitutes the anterior and posterior wings of the flap [8]. There is an area between the anterior and posterior wings of the flap, where the soft tissue is attached to the retroauricular groove, which acts as the central shaft. The arterial arcade comes from branches of the posterior auricular and superficial temporal arteries in the retroauricular groove that supplies the flap [8]. By using the central shaft as an axis, the flap is rotated vertically $180^{\circ}$ and transferred to the anterior auricular defect area tthrough the window created by removal of the auricular cartilage. The flap conceals the defect and primary closure of the flap and donor site with the adjacent skin is performed to complete the reconstruction.

In this case, we modified the advancement flap and reconstructed the defect using a simple method. The flap used for reconstruction was harvested only from the postauricular area. In addition, unlike the postauricular island flap, the flap was advanced from postauricular area to the defect in the cavum concha, which simplifies the procedure to perform. In addition, incision of the skin at the auricular slit passing area made the flap like an island flap. By making an incision, the flap passing through the auricular cartilage could be sutured and closed without any defect. Therefore, our advancement flap is easier to perform than the postauricular island flap and requires lesser operative time.

Postauricular island flaps have advantages such a skin color, texture, and thickness match, one-stage reconstruction, ability to conceal the donor site deformity, and satisfactory esthetic as well as functionally $[7,9]$. By this technique, the defect in the cavum concha can be concealed relatively well, while ensuring sufficient soft tissue in the postauricular area for primary closure.

The disadvantages of the postauricular island flap include risks such as pinning of the ear, chondritis, infection, the possible need for a postauricular drain, and necrosis [10]. In our case, compared to the postauricular island flap, we preserved the auricular cartilage by creating a slit and using less skin for the flap. Therefore, our technique ensure better esthetic outcomes by preserving the original auricular shape and causing lesser changes in the donor site.

In this case, the flap showed ischemic changes after 2 days because of the compression caused by the narrow slit. There were no further ischemic changes after releasing the flap by widening the slit for sufficient blood supply. An important factor during reconstruction to ensure adequate blood supply is creating a sufficiently large slit window that does not block the blood flow by removing sufficient auricular cartilage.

We planned a second-stage surgery to minimize skin defects and to ascertain negative surgical margins considering our lack of clinical experience. Generally, a one-stage surgery can be performed and better surgical outcomes can be expected.

We performed a modified advancement flap procedure to reconstruct the anterior auricular defect caused by resection of the CSCC and achieved excellent cosmetic and surgical outcomes. Therefore, we suggest that our technique can be an option for reconstruction of defects in the auricle. 


\section{Conflicts of interest}

The authors have nothing to disclose.

\section{References}

1. Oh CM, Cho H, Won YJ, Kong HJ, Roh YH, Jeong KH, et al. Nationwide trends in the incidence of melanoma and nonmelanoma skin cancers from 1999 to 2014 in South Korea. Cancer Res Treat 2018;50:729-37.

2. Gurudutt VV, Genden EM. Cutaneous squamous cell carcinoma of the head and neck. J Skin Cancer 2011;2011:502723.

3. Humphreys TR, Goldberg LH, Wiemer DR. The postauricular (revolving door) island pedicle flap revisited. Dermatol Surg 1996;22:148-50.

4. Brodland DG, Zitelli JA. Surgical margins for excision of primary cutaneous squamous cell carcinoma. J Am Acad Dermatol 1992;27(2 Pt 1):241-8.
5. Son SB, Seo SH, Kim IH. Original article: clinical findings and recurrence rate of 123 skin cancers treated with Mohs microscopic surgery. Korean J Dermatol 2008;46:8-15.

6. Masson JK. A simple island flap for reconstruction of conchahelix defects. Br J Plast Surg 1972;25:399-403.

7. Dessy LA, Figus A, Fioramonti P, Mazzocchi M, Scuderi N. Reconstruction of anterior auricular conchal defect after malignancy excision: revolving-door flap versus full-thickness skin graft. J Plast Reconstr Aesthet Surg 2010;63:746-52.

8. Golash A, Bera S, Kanoi AV, Golash A. The revolving door flap: revisiting an elegant but forgotten flap for ear defect reconstruction. Indian J Plast Surg 2020;53:64-70.

9. Jackson IT, Milligan L, Agrawal K. The versatile revolving door flap in the reconstruction of ear defects. Eur J Plast Surg 1994;17:131-3.

10. Krespi YP, Pate BR Jr. Auricular reconstruction using postauricular myocutaneous flap. Laryngoscope 1994;104(6 Pt 1):778-80. 